Table 1

Mean Trials to Criterion on the Second Problem

\begin{tabular}{ccc}
\hline Experiment & $100 \%$ Group & $50 \%$ Group \\
\hline 1 & 10.7 & $17.8^{*}$ \\
2 & 7.6 & $16.8^{* *}$ \\
3 & 19.6 & $34.0^{* *}$ \\
\hline
\end{tabular}

*Not significant

domain of complex hypotheses for the solution to Problem 2. Subjects in the $100 \%$ group received a simple first problem, which tended to direct them to search for simple solutions to the next problem. Since the solution to Problem 2 was, in fact, from the simple domain, the $100 \%$ group solved relatively quickly, whereas the $50 \%$ group was hindered.

\section{REFERENCES}

Bower, G., \& Trabasso, T. Concept Identification. In R. C Atkinson (Ed.), Studies in mathematical psychology. Stanford: Stanford University Press, 1963.

Humphreys, L. G. Acquisition and extinction of verbal expectation in a situation analagous to conditioning. Journal of Experimental Psychology, 1939, 25, 284-301.

Levine, M. Hypothesis behavior by humans during discrimination learning. Journal of Experimental Psychology, 1966, 71, 331-338.

Levine, M. A transfer hypothesis, whereby learning-to-learn, Einstellung, the PREE, reversal-nonreversal shifts, and other curiosities are elucidated. In R. L. Solso (Ed.) Theories in cognitive psychology; The Loyola symposium, Lawrence Erlbaum Associates, Potomac, Maryland, 1974.

Meyers, L. S., Dreissen, E., \& Halpern, J. Transfer following regular and irregular sequences of events in a guessing situation. Journal of Experimental Psychology, 1972, 92, 182-190.

(Received for publication October 1, 1974.)

Bulletin of the Psychonomic Society

1975, Vol. 5 (1), 76-78

\title{
Resistance to extinction in humans as a function of sequential variables and amount of acquisition training
}

\author{
R. SCHMECK and C. MUELLER \\ Southern Illinois University, Carbondale, Illinois 62901
}

\begin{abstract}
The experimenters used human subjects and three schedules of reinforcement: (1) continuous reinforcement; (2) a schedule with few N-R transitions and long N-lengths; and (3) a schedule with many N-R transitions and short N-lengths. In the first experiment, with extended acquisition training, the second schedule produced a partial reinforcement effect, but the third did not. In the second experiment, acquisition training was shortened, but the results were essentially the same as those of the first experiment.
\end{abstract}

Since the early demonstrations of partial reinforcement effects in human subjects (Humphreys, $1939 a, 1939 b)$, there have been relatively few studies investigating the effects of sequential variables on the phenomenon. Grosslight, Hall, and Murnin (1953) and Poon and Halpern (1971) demonstrated that a partial reinforcement schedule produces greater resistance to extinction in human subjects if it contains more trials on which the response is rewarded immediately following trials on which the response is not rewarded (N-R transitions). In addition, Meyers and Capaldi (1970) and Halpern and Poon (1971) determined that the resistance to extinction of human subjects was increased if they received more consecutive nonrewarded trials prior to rewarded trials (N-length) (i.e., schedules which gave subjects more experience with longer N-lengths produced greater resistance to extinction).

When studying the effect of N-R transitions and $\mathrm{N}$-length, one encounters a problem inherent in attempting to vary one sequential variable while holding the other constant. Variation in one of the variables tends to produce simultaneous variation in the other.
Grosslight et al. (1953) attempted to vary N-R transitions while holding $\mathrm{N}$-length constant by giving the group that was to receive no $\mathrm{N}-\mathrm{R}$ transitions a brief rest period following each series of nonrewarded trials. However, the authors point out that these subjects might well have retained the experience of nonreward during the rest period. Thus, they would have had the subjective experience of an N-R transition when the reinforcement schedule was resumed following the rest period.

The present studies varied N-R transitions and N-lengths simultaneously. Capldi (1967) has indicated that, when rats are used as subjects, the impact of sequential variables is related to the number of training trials given prior to extinction. When subjects are given relatively extended training (i.e., when asymptotic habit strength is reached), N-length is more frequently the major determiner of resistance to extinction. This prediction was supported by Halpern and Poon (1971) using extended training, but no continuous reinforcement control group was included. The first study reported below gave subjects extended training 
(26 trials) with two different 50\% reinforcement schedules. One schedule contained many N-R transitions and short N-lengths; the other contained few N-R transitions and long $\mathrm{N}$-lengths. In addition, a continuous reinforcement control group was run. It was anticipated that both schedules would produce a partial reinforcement effect when compared to the continuous reinforcement control group, but that the schedule with longer $\mathrm{N}$-lengths would yield the greatest effect since extended training was given to the subjects.

Several studies (e.g., Halpern \& Poon, 1971; Meyers \& Capaldi, 1970), using human subjects in partial reinforcement research, have incorporated procedures that deviate in important ways from the instrumental conditioning procedures employed with rats as subjects (e.g., Capaldi, 1964). Specifically, human subjects have frequently been asked to predict whether or not a light would come on during a particular trial or which of two or more lights would come on, i.e., they were given a choice between response alternatives ("yes, the light will come on" and "no, the light will not come on"). The problem with this procedure is that it involves several different response-reinforcement contingencies (e.g., "yes it will"-light; "no it won't"-light; "yes it will"-no light; "no it won't"-no light). The present study did not give subjects such a choice. It is true that they could have chosen between responding and not responding, but the procedure was such that, if no response was made, subjects were given no feedback at all (just as the rat that fails to negotiate the runway has no way of determining whether there was food in the goalbox). Also, the instructions were such as to produce virtually $100 \%$ responding, as in the runway studies with rats. Likewise, in the present study, the reinforcement schedule was linked to the responses of the subject rather than the trial number, again paralleling the rat studies.

\section{EXPERIMENT I}

\begin{abstract}
Method
Thirty-six introductory psychology students, 18 males and 18 females, were randomly assigned to three groups (12 per group) with the restriction that each group be assigned half males and half females. The apparatus was a modified Humphreys board with the control panel situated in another room behind a one-way observation mirror. The subject's console contained two lights, one round and small in diameter and the other square and slightly larger, mounted horizontally $7 \mathrm{~cm}$ apart. Fifteen centimeters below the square light was a large red button. The subject's task was to attempt to turn on the square light by pushing the button each time that the round light was illuminated. Twenty-six acquisition trials were presented to all subjects with a different $50 \%$ schedule of reinforcement employed in each group. The schedules were NNNNRRRRRNNNNNRRRRRNNNNRRR (mean N-length $=4.33 ; \mathrm{N}-\mathrm{R}$ transitions $=3$ ) and NNRRNNNRRRNNRRNNNRRRNNNRRR (mean N-length $=2.60 ; \mathrm{N}-\mathrm{R}$ transitions $=5$ ). A control group was also run using a continuous reinforcement schedule for 26 trials.
\end{abstract}

Subjects were run individually and told to imagine that they were working on an assembly line. They were told that their task was to turn on the square light, by pushing the button once whenever the small round light was illuminated. If the square light came on after the button had been pushed, the machine had supposedly functioned properly. Subjects were instructed that the management knew that the machine might function unreliably (i.e., the square light might not always come on when the button was depressed), but the subjects were told to continue pushing the button as long as the machine was working at all. Subjects were also instructed that the machine would, in fact, break down at some point in the experiment, and that part of their job was to determine when this had occurred. This was done in order to eliminate the tendency of some subjects to respond for inordinate periods of time when feedback is terminated. They were told that six consecutive nonresponses in the presence of the illuminated round light would be taken as an indication that they believed that the machine had broken down. The six consecutive nonresponses also served as the extinction criterion but only if they occurred during the actual extinction trials. That is, if the square light had not, in fact, been permanently shut off, then the onset of the round light following the six nonresponses would indicate to the subject that he was to resume responding since he had incorrectly assumed that the machine was broken. Fortunately, this occurred only one time for one of the subjects (i.e., as noted above, responding during acquisition was virtually $100 \%$ ). The measure recorded was the number of button presses made in the presence of the illuminated round light during the extinction phase of the experiment (i.e., after the square light had been permanently shut-off).

\section{Results and Discussion}

Analysis of variance indicated a significant treatment effect $[\mathrm{F}(2,33)=6.69, \mathrm{p}<.005]$. As expected subsequent analyses (Newman-Keuls) indicated a significant difference between the mean trials to extincton for the long N-length/few N-R group (9.92) and the short N-length/many N-R group (6.75) $(\mathrm{p}<.05)$. Also, analysis indicated a significant difference between mean trials to extinction for the long $\mathrm{N}-$ length/few N-R group and the control group (5.42) $(p<.01)$. Surprisingly, the difference between the short $\mathrm{N}$-length/many N-R group and the control group was not significant. It was originally expected that both groups would show a partial reinforcement effect with the effect being greater in the group that received the longer $\mathrm{N}$-lengths. Also, the relationship between these results and those of Halpern and Poon (1971, Experiment III) is not clear, since the latter study did not include a continuous reinforcement control group. Thus, the second study was designed to be an essential replication of the first with the exception that fewer acquisition trials were administered. On the basis of Capaldi's (1967) assumption that short acquisition training (and preasymptotic habit strengths) would cause N-R transitions to be the main determiner of resistance to extinction, it was predicted that either (1) the short $\mathrm{N}$-length/many N-R group would produce the greater resistance to extinction, as was the case in the Poon and Halpern (1971) study, or (2) the partial reinforcement effect of the short N-length/many N-R group would at least reach significance under these conditions. 


\section{EXPERIMENT II}

\section{Method}

The method (including selection and assignment of subjects) used in this experiment was the same as that in the first one except that acquisition training consisted of 10 rather than 26 trials. The $50 \%$ schedules employed were NRRNRNNRNR (mean N-length $=1.25 ; \mathrm{N}-\mathrm{R}$ transitions $=4$ ) and NNNRRRRNNR (mean N-length $=2.50 ; \mathrm{N}-\mathrm{R}$ transitions $=2$ ). $\mathrm{A}$ control group was also run, using a continuous reinforcement schedule for 10 trials.

\section{Results and Discussion}

Analysis of variance once again indicated a significant treatment effect $[F(2,33)=12.89, p<.001]$. Subsequent analysis (Newman-Keuls) revealed a significant difference between the mean trials to extinction for the long N-length/few N-R group (13.50) and the short $\mathrm{N}$-length/many $\mathrm{N}-\mathrm{R}$ group $(7.67)(\mathrm{p}<.01)$. Analysis also indicated a significant difference between trials to extinction for the long N-length/few N-R group and the control group (6.08) $(\mathrm{p}<.01)$. However, the difference between the short N-length/many $\mathrm{N}-\mathrm{R}$ group and the control group was, once again, not significant. Surprisingly, the long N-length/few N-R group was again more resistant to extinction than the short $\mathrm{N}$-length/many $\mathrm{N}-\mathrm{R}$ group. In addition, the latter experimental group again failed to show a partial reinforcement effect.

\section{CONCLUSIONS}

In the first study, it was anticipated that extended acquisition training would cause the reinforcement schedule with the longer N-length to produce greater resistance to extinction, as one would expect given Halpern and Poon's (1971) results. Although the schedule with the longer N-lengths did produce greater resistance to extinction, the results failed to show any partial reinforcement effect for the group that received short N-lengths coupled with many N-R transitions. As noted earlier, it is difficult to compare this result with Halpern and Poon (1971, Experiment III) since the latter study did not include a continuous reinforcement control group. Thus, a second study was designed employing fewer training trials. Based on Capaldi's (1967) analysis of the interaction of sequential variables and amount of training in rats, one would suspect that reducing the number of trials might increase the impact of N-R transitions. However, subjects in the second study again failed to show a partial reinforcement effect when the short N-length was coupled with many N-R transitions. Also, it seems unreasonable to suggest that the anticipated effect would have occurred in the second study if fewer than 10 trials had been used, since the absolute superiority of the long $\mathrm{N}$-length schedule was even greater in the second study than in the first, i.e., there was no trend toward a reversal of the position of the two experimental groups.

There is a disturbing contrast between the results of the second study described above and the one conducted by Poon and Halpern (1971). The latter study was very similar to the present one but obtained superior resistance to extinction in the short N-length/many N-R group. The most distinctive difference between the procedures of the two studies is the fact that the present experiment clearly informed subjects of the eminent extinction phase (i.e., they were supposed to determine when the "machine had broken down"). In the Poon and Halpern (1971) study, subjects did not know that the apparatus would ever cease to function. The importance of this procedural difference is pointed up by the fact that groups in the Poon and Halpern (1971) study were from four to six times more resistant to extinction than subjects in the present studies. It may well be the case that when individuals are instructed to detect the termination of feedback (e.g., a quality-control task in industry) the number of successive no-feedback trials (rather than the number of times that feedback trials follow no-feedback trials) is the major determiner of when the observer will report a "breakdown".

Finally, a comment should be made regarding the apparently greater resistance to extinction obtained in the second study. Since the amount of time required to run 10 acquisition trials was only 2 min, subjects may have been hesitant to assume that the experiment was drawing to a close after such a brief period of time. If this was the case, the overall number of responses to extinction in the second study would be somewhat inflated.

\section{REFERENCES}

Capaldi, E. J. Effect of N-length, number of different N-lengths, and number of reinforcements on resistance to extinction. Journal of Experimental Psychology, 1964, 68, 230-239.

Capaldi, E. J. A sequential hypothesis of instrumental learning. In K. W. Spence \& J. T. Spence (Eds.), The psychology of learning and motivation. New York: Academic Press, 1967.

Grosslight, J. H., Hall, J. F., \& Murnin, J. Patterning effect in partial reinforcement. Journal of Experimental Psychology, $1953,46,103-106$.

Halpern, J., \& Poon, L. Human partial reinforcement extinction effects: An information-processing development from Capaldi's sequential theory. Journal of Experimental Psychology Monograph, 1971, 89, 207-227.

Humphreys, L. G. The effect of random alternation of reinforcement on the acquisition and extinction of conditioned eyelid reactions. Journal of Experimental Psychology, 1939a, 25, 141-158.

Humphreys, L. G. Acquisition and extinction of verbal expectations in a situation analogous to conditioning. Journal of Experimental Psychology, 1939b, 25, 294-301.

Meyers, L. S., E. J. Resistance to extinction as a function of sequence of events in partial reinforcement. Psychonomic Science, 1970, 19, 199-200.

Poon, L., \& Halpern, J. A small-trials PREE with adult humans: Resistance to extinction as a function of number of $N-R$ transitions. Journal of Experimental Psychology, 1971, 91, 124-128.

(Received for publication September 17, 1974.) 